\title{
Microscope as a teaching tool in Fourier transform optics
}

\author{
Maksymilian Pluta
}

Maksymilian Pluta, "Microscope as a teaching tool in Fourier transform optics," Proc. SPIE 1603, Education in Optics, (1 March 1992); doi: $10.1117 / 12.57890$

\section{SPIE. Event: Education in Optics, 1991, Leningrad, Russian Federation}




\title{
Microscope as a teaching tool in Fourier transform optics
}

\author{
laksymilian Pluta \\ Institute of Applied Optics (formerly Central Laboratory of Optics) \\ Department of Physical Optics \\ ul.Kamionkowska 18, 03-805 Warsaw, Poland
}

\section{ABSTRACT}

In general, the microscope is a basic instrument of research, but it also may extensively be used for modern education in physical optics, and especially in Fourier transform optics. This can be shown in a quite new way using a polarizing microscope and birefringent fibers, which can produce a large number of known, less known and even unknown optical Fourier transforms.

\section{INTRODUCTION}

Althout the basic principles of image formation in the microscope within the scope of diffraction theory with some inclination to Fourier optics were formulated by E.Abbe in 1873 and then (1911 to 1920) refined mathematically by a Polish physicist, M.Wolfke, the theory's real potential was revealed some decades later when the phase contrast was discovered by F.Zernike $(1932-1935)$, and the Fourier transform was evidently applied to optics by P.M.Duffieux (1946).

This paper was originally prepared as a long presentation with a variety of problems and many illustrations. Its length must now be reduced to a minimum to present a contribution of reasonable space. Consequently, the title of the paper should read here as follows:

\section{POLARIZED-LIGHT MICROSCOPE and BIREPRINGEITT PIBERS \\ 28 TEACHIXG TOOLS II POURIER TRAMSFORY OPIICS}

Both polymer textile fibers and optical fibers guiding polarized light are taken into consideration. In fact, however, the former were already covered by at least three publications ( see Refs.1 to 4 ). On the other hand, birefringent optical fibers (Bow-Tie, Panda) were only preliminary examined some time ago ( see Ref.2), and then the author of this contribution has offered this problem to M.Boziyk affiliated with the Department of Physics, Technical University of Białystok, Poland. Her more systematic studies are carried out under a research project sponsored by the Ministry of National Education, and quite recently she prepared a preliminary scientific report on Fourier transforms of a variety of birefringent optical fibers (see Ref.5).

\section{EXPERIMENTAL APPARATUS}

A standard, but slightly modified, polarizing microscope can be used for producing the optical Fourier transforms of birefringent fibers (Figure 1). The modification consists in adding a slit subcondenser diaphragm $D$, linear continuous interference filter IF and a double-refracting wollaston prism W. The prism Whowever, is not necessary for observation of the optical Fourier transforms of birefringent fibers $\mathrm{F}$, but serves only for measuring light wavelengths $\lambda$ in 
real time. This is achieved via a calibration plot $b(\lambda)$, where $b$ is the period of the fringe interference pattern produced by the Wollaston prism in monochromatic light and observed in the image plane $\Pi^{\circ}$ of the microscope objective ob. Monochromatic light is extracted from a microscope halogen lamp (not shown in Fig.1) and varied by transverse translation of the interference filter IF. Honochromatic light. of variable wavelength $\lambda$ was used for measuring birefrinbence.

$$
B=n_{11}-n_{1}
$$

of highly birefringent fibers (e.g.,polymer textile fibers; see Ref.t for details). Here $n_{\mid l}$ and $n_{\perp}$ are the refractive indices of the fiber for light components vibrating parallel $(11)$ and perpendicular $(\perp)$ to the fiber axis.

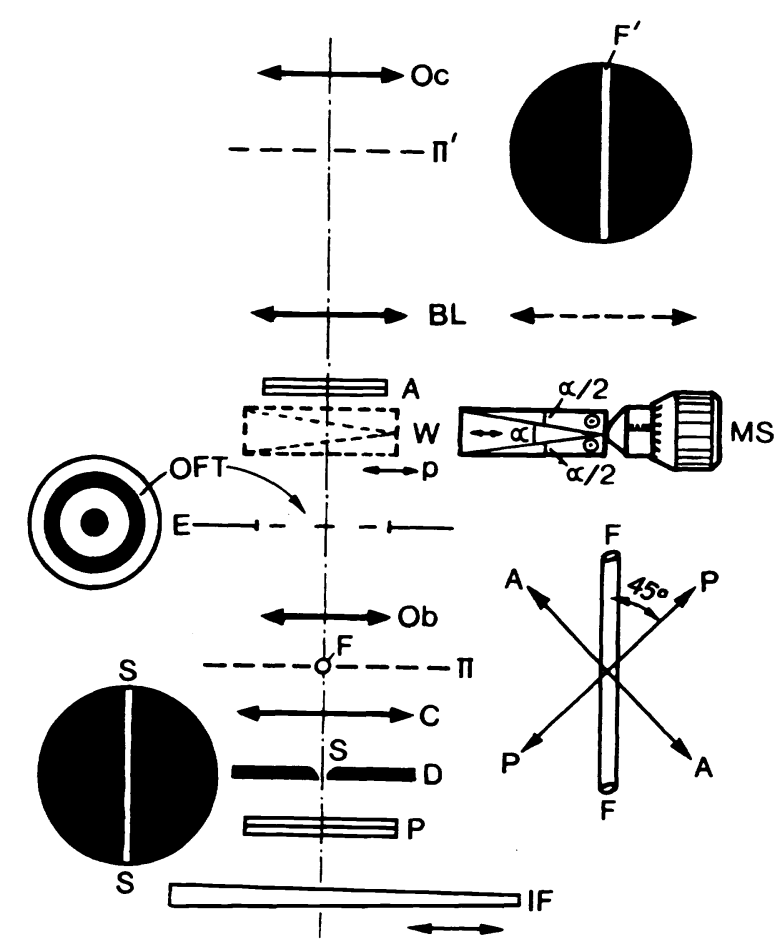

Fig.1. Schematic diagram of a polarizing microscope system used for observation and processing of the OFTs of birefringent fibers.
As shown in Figure 1 , the stit diaphragm $D$ is located in the front focal plane of the substage condenser $C$, thus light. incident on the birefringent fiber $P$ consists of parallel beams. Optical Fourier transforms (OFT) are observed, via a microscope ocular $O C$ and Bertrand lens $B L$, in the exit pupil or rear focal plane $\mathrm{E}$ of the objective $\mathrm{Ob}$. The lens $\mathrm{BI}$ can be removed from the path of light, and a normal microscopical image $F$ of the fiber $F$ under study arises in the image plane $\Pi^{\circ}$ and is observed through the ocular Oc alone. If simultaneously the Wollaston prism $W$ is inserted into the path of light, a fringe interference pattern is also observed in the plane $I$ The interfringe spacing $b$ is measured by means of the micrometric screw associated with the transverse $(p)$ movement of the prism 1 .

The basic orientation of the polarizer $P$, analyzer $A$ and slit $S$ of the condenser diaphragm $D$, and of the fiber $F$ under study are shown in the left-hand and right-hand sides of the diagram (Fig.1), where SS denotes the direction of the condenser slit S, $P P$ and $A A$ are the directions of light vibration (axes) of the polarizer $P$ and analyzer $A$, respectively, and $F F$ is the direction of the fiber axis.

\section{BIREFRINGENT FIBER AS A BIFOCAL CYLIIDRICAL LENS}

When surrounded by a medium of refractive index $n^{*}$, a birefringent fiber oriented diagonally between two crossed polars ( $P$ and $A$ as shown in Fig. 1 ) acts as a specific bifocal cylindrical lens ( see Fig.2), whose focal lines $I_{1} /$ and $L_{1}$ contain light vibrations parallel. ( 11$)$ and perpendicular $(1)$ to the fiber axis. The focal lengths $f_{1} l$ and $f_{\perp}, i . e .$, the distances between the fiber center and the focal lines, are defined by 


$$
f_{11}=2 x \frac{n_{11}}{4\left(n_{11}-n^{\circ}\right) n^{\circ}} \quad \text { and } \quad f_{1}=2 x \frac{n_{1}}{4\left(n_{1}-n^{\circ}\right) n^{\circ}}, \quad \text { (2) }
$$

where $2 \mathbf{r}$ is the fiber diameter.

Depending on the refractive index $\mathrm{n}^{\prime}$, the fiber diameter $2 \mathrm{r}$ and the fiber

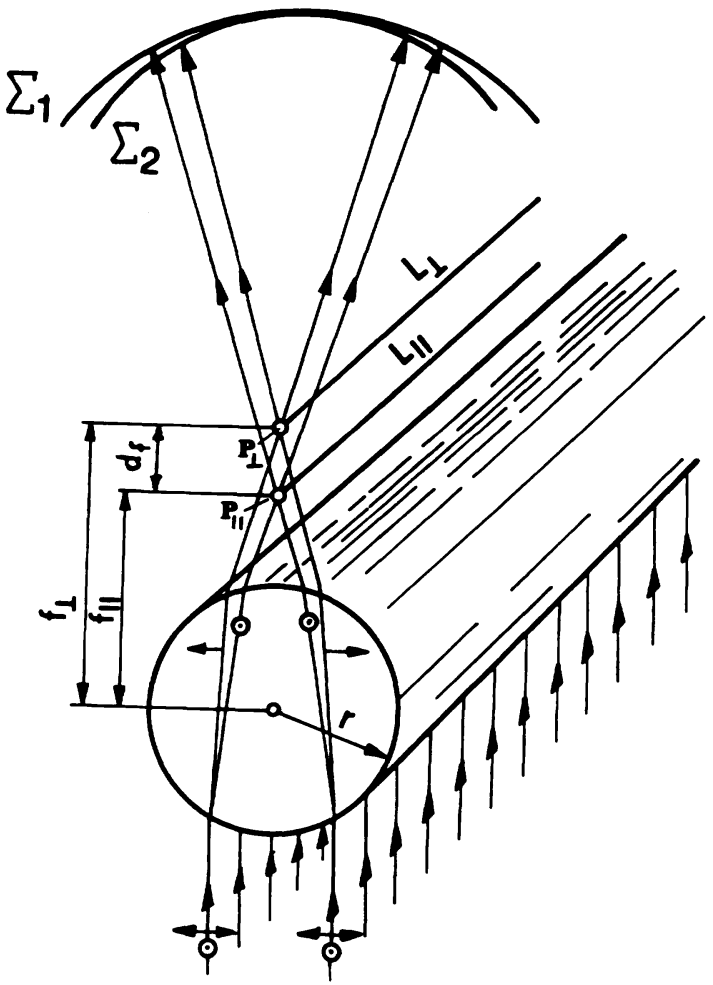

Fig.2. Birefringent fiber as a bifocal cylindrical lens. birefringence $B$, the focal lengths $f_{11}$ and $f_{1}$ can be greatly varled, and thus the fiber $F$ manifests itself as a single-, double- or multiple-slit source of light. Horeover, the doubleslit can be vertical, due to the focal lines $I_{l} \mid$ and $I_{\perp}$ separated vertically as shown in Pig.2, or horizontal. On the other hand, three or even rore slits line sources of light occupy a horizontal plane at right angles to the optic axis of the microscope abjective. In particular, such a qualification applies to polymer textile fibers made by spinning and drawing.

\section{OPI OP THE DIRAC DELTA FUNCTIOH}

This optical Fourier transform is observed when the fiber birefringence $B$ is very small. Consequently, the difference df between the focal lengths $f_{\perp}$ and $f_{\mid 1}$ (Fig.2),i.e.,

$$
d_{f}=f_{\perp}-f_{11}
$$

is also very small and the two focal lines $I_{11}$ and $I_{\perp}$ are practically unresolvable and manifest themselves as a

single focal line equivalert to a slit in an opaque screen( see Fig.3a) illuminated by a parallel light beam. If the focal line is extremely narrow, it can be

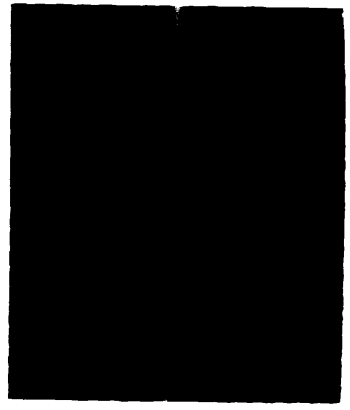

a)

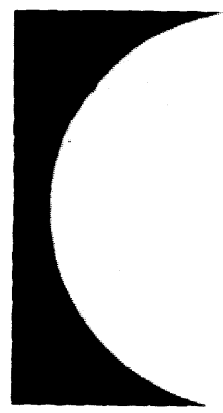

b)

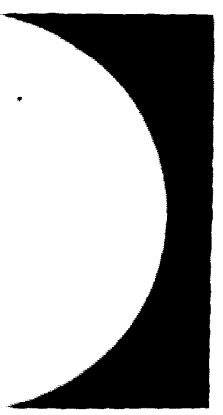

)

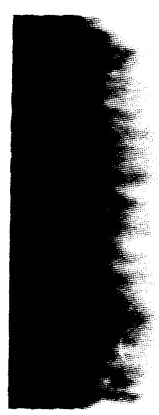

c)

Fig.3. Focal line (a), its OFT (b), and conventional image (c) of a weakly birefringent textile fiber surrounded by air medium. Objective magnifying power/ numerical aperture: $40 x / 0.65$. 
treated as the one-dimensional Dirac delta function, whose Fourier transform is equal to unity as shown in Fig. 3b. This means that the wavefront is a plane surface in the Fourier plane (rear focal plane) of the objectire (Ob, Fig.1) and has the same amplitude over the objective exit pupil $\mathrm{B}$. Such a situation as shown in Fig. 3 is usually produced by undrawn cylindrical polymer textile fibers, whose birefringence $B$ is normally weak and therefore permits us to obtatn excellent. Dirac delta functions if the fiber is surrounded by an air medium, while the condenser slit (S, Fig.T) is sufficiently narrow and exactly parallel to the fiber axis PF.

\section{OFT OF A GAUSS FUNCTION}

If a birefringent fiber that produces the Dirac delta function in an air medium (Fig.3a), is then immersed in a liquid whose refractive index " is much higher than unity, say, $n^{\circ}=1.3$ to 1.5 , the fiber focal line becomes wider ( $\mathrm{Pig} .4 \mathrm{a}$ ) and manifests itself as a Gauss function. Consequently, its OPT is aIso

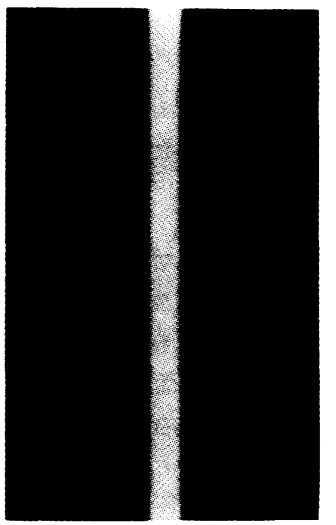

a)

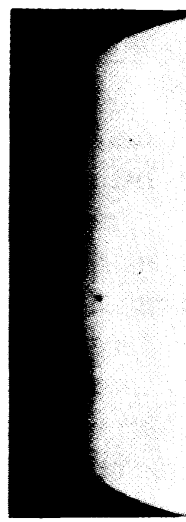

b)

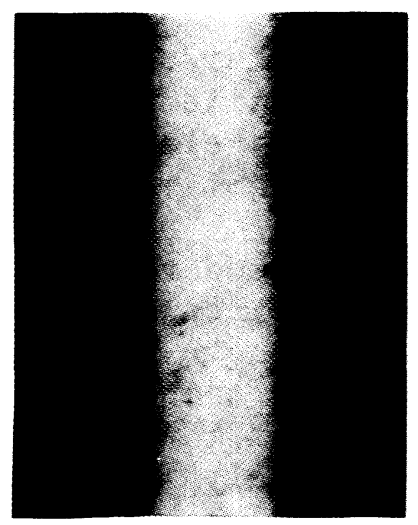

c)

Fig.4. As in Fig.3, but here the fiber is surrounded by a liquid medium of refractive index $n^{\circ}=1.515$

of the Gauss-function character (Fig.4b) across the focal line. This OFT, however, is less interesting and less useful in practice than that shown in Fig.3b.

\section{OPT OF THE VERTICAL DOUBLE-SLIT}

A cylindrical fiber whose birefringence $B$ is significant produces two focal lines $I_{I I}$ and $I_{I}$ (see Fig.2) separated from each other by a distance $\left.d_{f}\right\rangle \lambda$ when the fiber is surrounded by an air medium. Such a situation applies to most polymer textile fibers after their drawing. How, two focal lines act as two light slits, one of which follows the other. These lines are mutually coherent across their widths but they are incoherent along their lengths. Each pair of coherent paints $P_{1} l$ and $P_{\perp}$ ( Fig.2) produces spherical wavefronts $\Sigma_{1}$ and $\Sigma_{2}$ whose radii of curvature are slightly different at a given distance from the fiber. The two wavefronts can interfere with each other and produce an interference pattern with annular/circular fringes, such as shown in Fig.5, observed in the Fourier plane. Any other pair of coherent points along the focal lines $I_{\text {! }}$ and $I_{1}$ gives rise to an individual interference pattern identical with that produced by light wavefronts emerging from the points $P_{\mid l}$ and $P_{\perp}$. All individual interference patterns are mutually incoherent, they occopy the same position in the Fourier plane of the microscope objective and produce, by incoherent superposition, an intense 
resultant interference pattern of circular symmetry (Fig.5). This pattern does not change, of course, its position when the fiber $\mathrm{P}$ (Fig.t) is transversely or vertically translated in the path of light. This property results from a well known theorem which states that the rourier transform does not translate if the object under the Fourier transformation is translated.

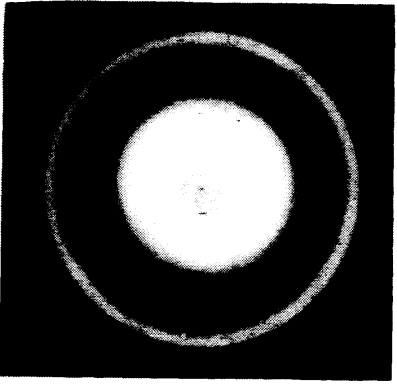

a)

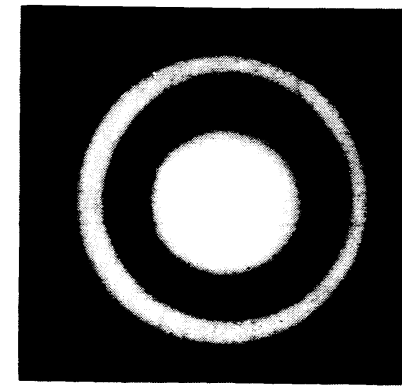

b)

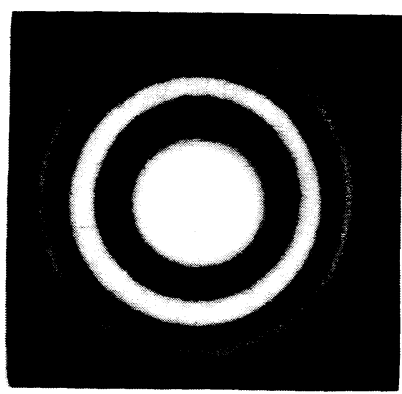

c)

Fig.5. Annular/circular OFTs produced by a highly birefringent polymer textile fiber surrounded by an air medium, with decreasing light welength from $\lambda \approx 570 \mathrm{~nm}(a)$ to $560 \mathrm{~nm}(b)$ and 540 $\mathrm{nm}(\mathrm{c})$. Objective magnifying power/numerical aperture:40x/0.65.

If the fiber is moderately birefringent,an OFT accurs which consists of a single annular or circular dark interference fringe. Its size and diameter depends on the wavelength $\lambda$ of monochromatic light used. The light wavelength can easily be varied by transverse sliding the interference filter IF as show in Fig.1. Starting from the long-wavelength region of the visible spectrum and approaching continuously to the short-wavelength spectral region permits us to observe a fascinating flow of the interference pattern and its annular fringes of consecutive order. Figure 5 shows almost a minor illustration of this phenomenon. Sometimes two annular dark fringes are simultaneously visible ( Fig.5c) in the exit pupil of a microscope objective of high numerical aperture if highly birefringent fiber is examined.

\section{WUTIPLE-SLIT OPTICAL FOURIER TRANSFORMS}

If a birefringent fiber is immersed in a liquid whose refractive index $n^{\circ}$ approximates the fiber refractive indices $n_{11}$ and $n_{\perp}$, then the focal lengths $f_{11}$ and $f_{\perp}$ and also the difference $d_{f}$ between the focal lines $I_{l, l}$ and $I_{\perp}$ (Fig.2) become long. No optical Fourier transforms of the focal lines are therefore observed instead, we can observe OPTs of the fiber itself. The cylindrical birefringent fiber now behaves as a multiple-slit object consisting of two, three, four, and even more parallel slits of various width arranged as a line grating in a plane perpendicular to the objective axis. In fact, these slit components are the bright interference fringes of polarized light. They are separated from each other by dark fringes. All these fringes are localized in or near to the object plane $\Pi$ of the objective $O b(F i g .1)$, and their widths and positions vary when the light wavelength $\lambda$ is changed.

Two fringe arrangements, however, are predominant: first, two relatively wide bright fringes, separated by a central dark fringe, appears for particular wavelengths ( $\mathrm{Fig.6 \textrm {G }}$ ); second, a wide bright fringe, surrounded by two dark fringes, covers the central zone of the fiber for other particular wavelengths of monochromatic light Fig.6c. In the first instance we observe an OFT similar to 
the Young interference pattern (Fig.6b), while in the second case the OFT( Fig. $6 d$ ) is similar to that of the squared sinc function. Very narrow bright fringes

a)

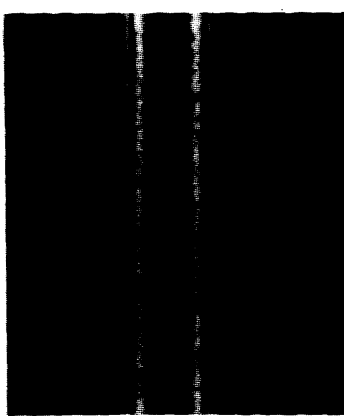

c)

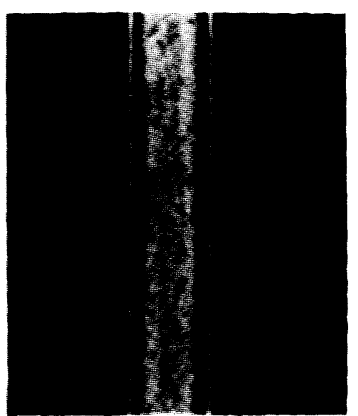

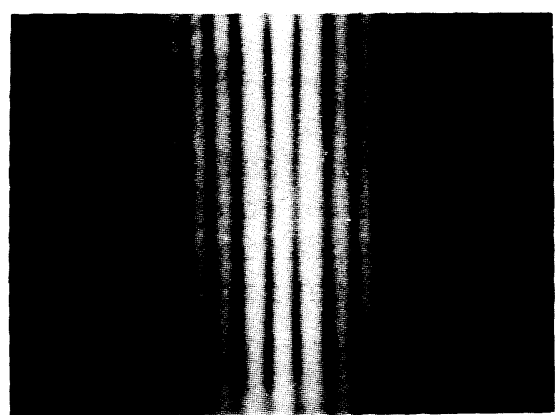

b)

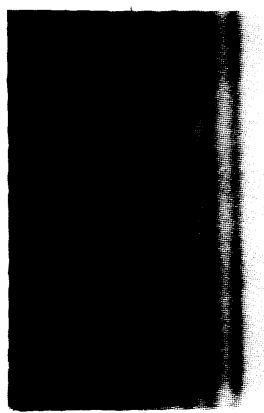

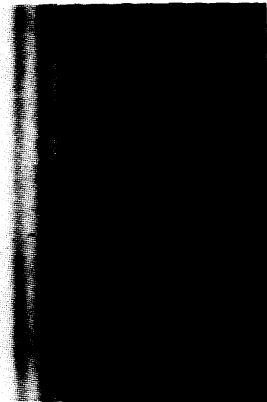

d)

Fig. 6. Microscopical images in polarized light (a) and ( c) and optical Fourier transforms $(b)$ and $(d)$ of a cylindrical birefringent textile fiber immersed in a liquid medium whose refractive index $n$ is near to the refractive indices $n_{I l}$ and $n_{\perp}$ of the fiber.

that are perceired at the marginal zones of the fiber images (Figs.6a and $6 \mathrm{c}$ ) contribute only slightly to the distribution of light intensity in the Fourier plane (Figs.6b and 6d).

\section{OPR OP ELLIPTICAL SHAPE}

If a polymer textile fiber is immersed in a liquid (e.g.,water) whose refractive index $n^{\prime}$ is much smaller than $n_{l l}$ and $n_{\perp}$, then the focal lengths $f_{l l}$ and $f_{\perp}$ (see Fig.2) are relatively long, but the difference $d_{f}$ between them is small

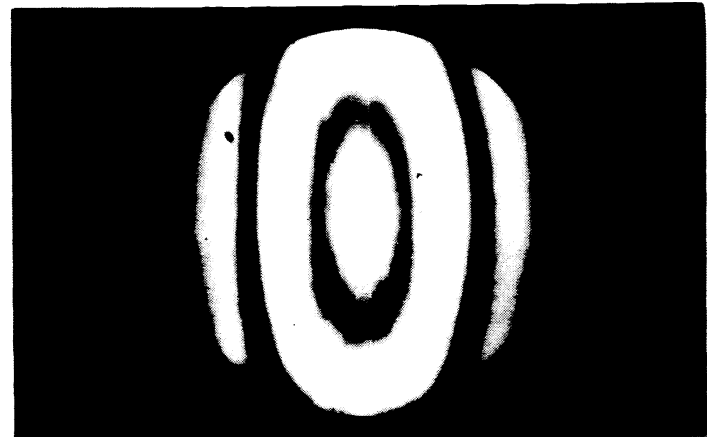

Fig.7. OFT pattern whose interference fringes are elliptical (typicaliy equal to several $\mu \mathrm{m}$ ). The OFTs that arise in such a situation are shom in Fig.7. The nature of their elliptical fringes cannot be as easily interpretated as that of interference patterns whose fringes are of annular/circular symmetry as shown in Fig.5. At any rate, this specific elliptical interference pattern is produced by both the fiber focal lines and the fiber itself and can be considered as an intermediate pattern between the annular and straight-line fringe patterns shown in Figs.5 and 6 , respectively. The practical usefulness of elliptical oPt patterns is nearly the same as that of the annular/circular OFT patterns. 


\section{OFIS OP OPTICAL PIBBRS}

Birefringent optical fibers are produced in another, more complicated way than polymer textile fibers. The "architecture" of the former greatly differs from that of the latter. Horeover, birefringence B( see Bq. (1)) of the optical fibers is typically much smaller than that of polymer textile fibers. Consequently, they produce the OFTs: which resemble roughly that of the Dirac delta function and Gauss function more or less modified by structural components (core, cladding, stress insertions) and their geometry. Some examples are shown in Figs.8 and 9.

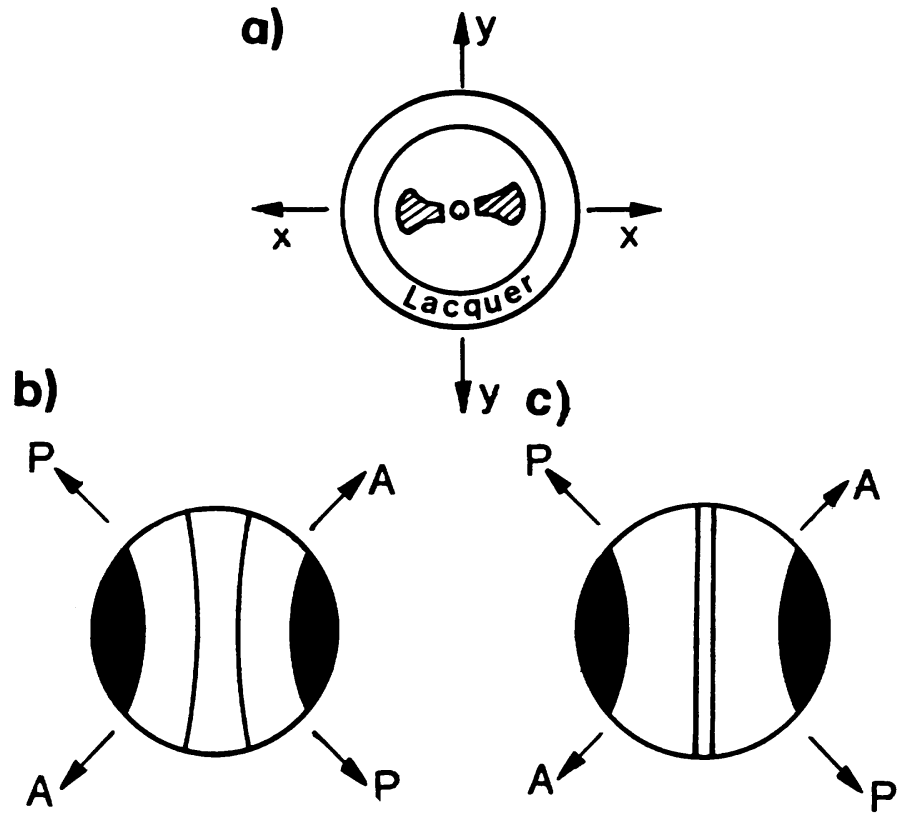

d)

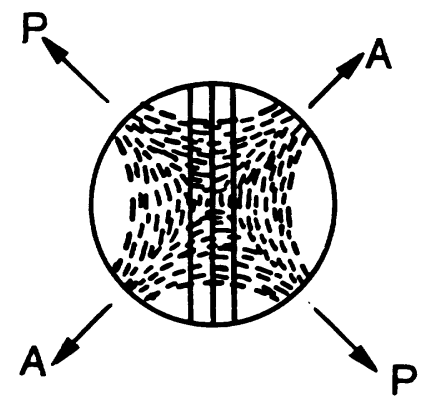

e)

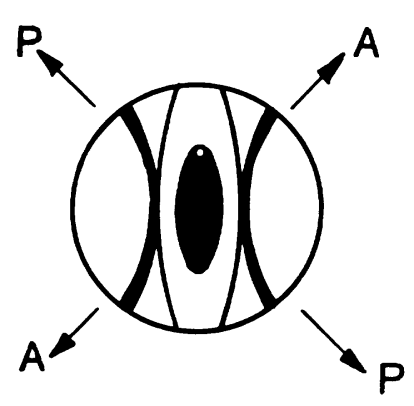

a)

b)

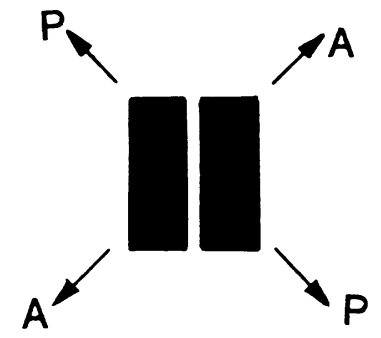

d)

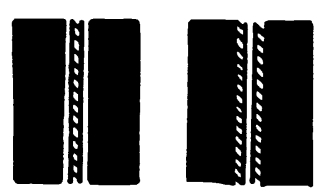

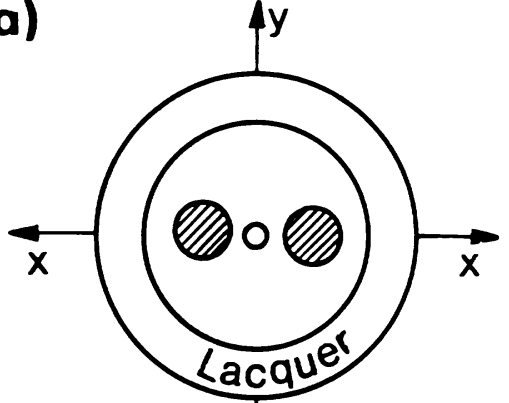

c)

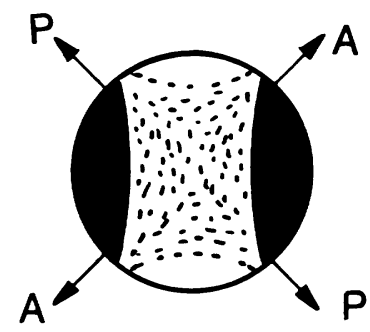

f)

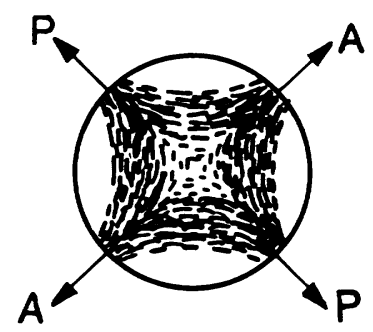

Fiz.8. Four ( $b, c, d$ and $e$ ) of many forms of the OFTs of a Bow-Tie (York) optical fiber whose cross-section is represented by diagram $a$. The OrTs $b$ and $c$ refer to the fiber with its lacquer layer, while $d$ and $e$ to the same fiber but free from its external lacquer layer. Objective magnifying power/numerical aperture: $40 \times / 0.65$.
Tig.9. This schematic diagram refers to an optical fiber Panda: al its crosssection, b), d) and e) conventional mfcroscopical images, c) and f) OPTs. The fiber is with its surrounding lacquer layer for diagrams $b$ and $c$, and free from this layer for diagrams do $f$. objective magnifying power/numerical aperture: $40 x / 0.65$. 
When a birefringent optical fiber, say, Bow-Tie (Fig.8) or Panda (Fig.9) is rotated about its own axis, then its OFT changes and the conoscopic (Maltese) cross can appear as a typical phenomenon observed in the exit pupil of the microscope objective of high numerical aperture if the polars ( $P$ and $A, F i g .1$ ) of the polarizing microscope are crossed. The conoscopic cross may easily be removed by turning slightly the polarizer P(Pig.1) or analyzer A from its ideal crossed position. Such an OPT free from the cross in question is shown in Pig. 10 .

It is, however, interesting to note that no conoscopic cross is observed when OFTs of polymer textile fibers are observed (see Figs. 3 to 7 ).

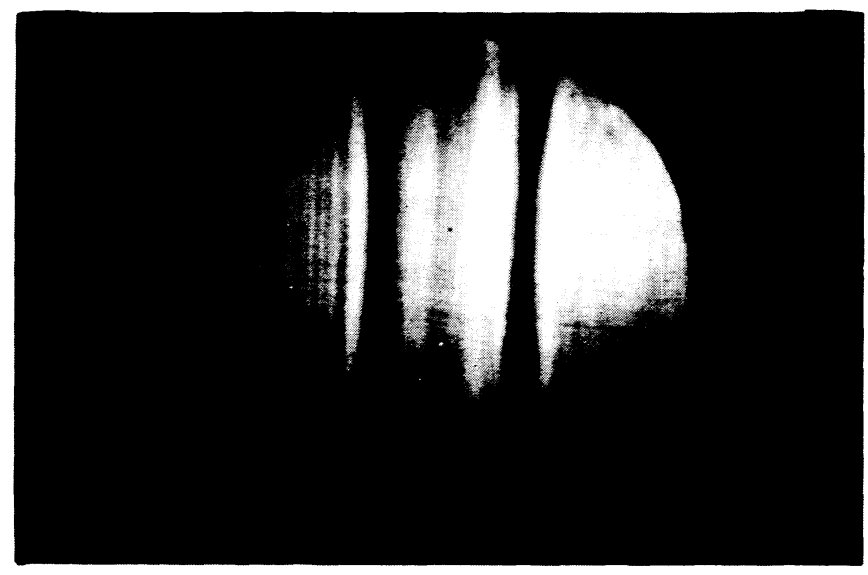

Fig.10. As in Fig.9f, but the conoscopic cross is removed ( Fhoto by courtesy of M.Bożyk, Technical University of Białystok).

\section{APPIICATIORS}

The birefringent fibers which produce extremely narron focal lines whose OFT is that of the Dirac delta function (see Fig.3) permitted us to develop a simple

a)
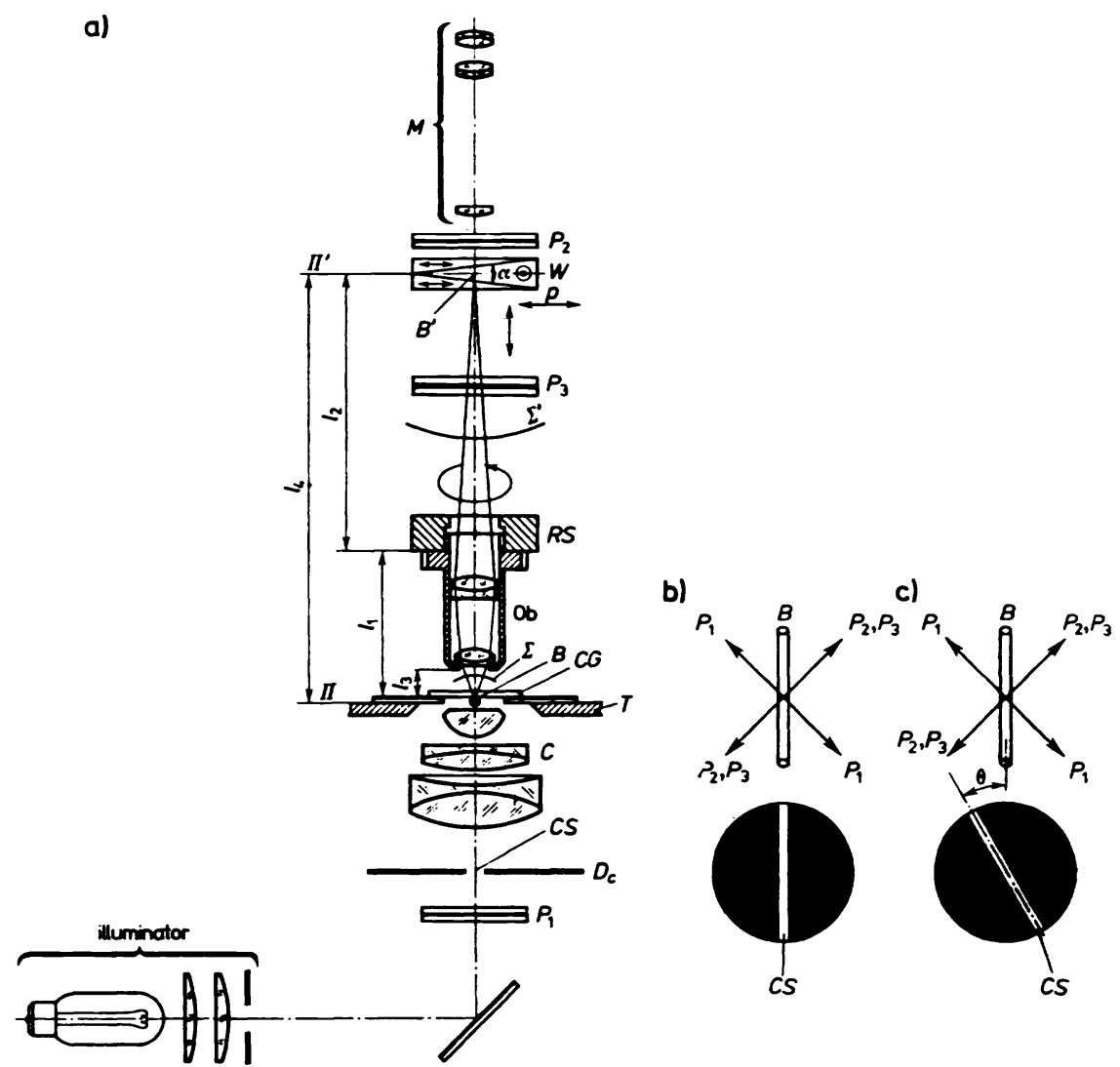
$M=$ auxiliary microscope of low power focused on the Fourier plane of the micros- cope objective ob under exa- mination,
$P_{1}, P_{2}$ and $P_{3}=$ polarizers, W double-refracting prism (symmetric Wollaston), II' = image plane of the ob- jective $\mathrm{Ob}$, $B^{\prime}=$ image of the focal line of the birefringent fiber $B$, $\Sigma$ and $\Sigma^{\prime}=$ wavefronts, RS = rotatable socket, $C G=$ cover glass for which the objective ob is correct- ed, $\Pi=$ object plane of the ob- jective $\mathrm{Ob}$, $\mathbf{T}=$ mechanical stage $\mathrm{x}, \mathrm{y}$, $C=$ condenser, $\mathrm{D}_{\mathrm{c}}=$ slit diaphragm,

Fig.11. Double-refracting interferometer which uses a birefringent fiber( $B$ ) whose OFP is equivalent to that of the Dirac delta function. 
double-refracting interferometer with variable direction of tilt of laterally sheared wavefront for testing microscope objectives. The optical system of this interferometer is shown in Fig.11.

As can readily be seen, this interference system is similar to that shown in Fig.1, but the Wollaston prism $W$ is positioned in the image plane $\Pi^{\prime}$ of the objective $O b$ to be tested, and an additional polarizer $\mathrm{P}_{3}$ is installed between the objective $\mathrm{Ob}$ and the Wollaston prism $W$. Horeover, the condenser slit CS is rotatable around the optic axis of the condenser $C$.

When the slit CS is exactly parallel to the birefringent fiber $B$ (Fig.11b) and the tested objective $O b$ is ideally free from aberrations and optimally focused on the fiber focal line, then the interference pattern in the Fourier plane looks like that shown in Fig.12a; no interference fringes occur and the area where the sheared wavefronts overlap is uniform in brighness (or homogeneous in tint if white light is used). If,however, the condenser slit CS (Fig.11)

a)
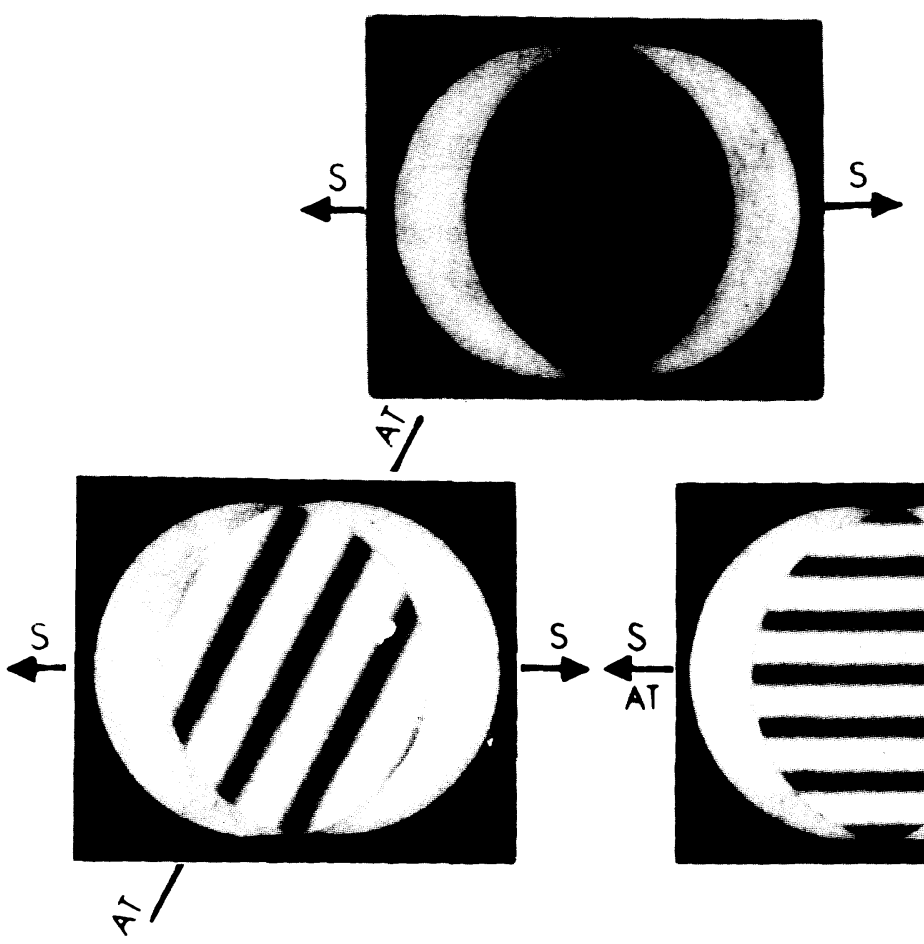

b)

c)

Fig.12. Exactly focused shear-interference images of an aberration-free microscope objective, illustrating the double-refracting interferometer for testing microscope objectives (Fig.11), which uses a birefringent fiber for producing the variable direction of tilt of laterally-sheared wavefronts.

forms an angle $\theta$ with the axis of the birefringent fiber $B(F i g .11 c$ ), then the uniform-field interference disappears and straigh-line fringes occur in the overlapping area of the sheared wavefronts (Fig.12b) and form an angle with the direction of wavefront shear S. For a particular value of $\theta$ the interference fringes become parallel to $S$, i.e. the axis of wavefront tilt,AT, is parallel to the direction of wavefront shear $S$ as shown in Fig.12c. 
Such a variable tilt of interfering wavefronts cannot be obtained if a conventional slit (e.g. a slit ruled in a metallic thin film evaporated onto a glass slide) is used instead of the birefringent fiber $B$ as show in Fig.11 (the conventional object slit does not require an additional polarizer $\mathrm{P}_{3}$ in the interference system shown in Fig.11, and the rotatable subcondenser slit CS becomes useless).

The use of the birefringent fiber, whose focal line is described by the Dirac delta function, radically improves the ability of the interferometer of this kind. First of all, its sensitivity is very high and the interpretation of the lateral shearing interference fringe patterns is easier when the tilt axis $1 T$ (Fig.12c) is parallel to the direction $S$ of wavefront shear.

The interference patterns such as shown in Fig. 12 correspond to those produced by an ideal microscope objective. Otherwise, if the objective suffers from a wave aberration, no uniform-field interference (Fig.12a) and no straight-line interference fringes (Figs.12b and $c$ ) can be obtained. A more detailed description of this interferometer can be found in Refs.3 and 4.

OFTs of circular/annular shape (see Pig.5) and those with elliptical interference fringes ( Fig.7) were applied to the measurement of the fiber birefringence B and its spectral dispersion. Especially, the annular/ circular ofts are very suitable for this application due to the fact that such optical Fourier transforms are produced by most polymer textile fibers surrounded by an air medium. There is no place to discuss this matter here and the reader is referred to Ref. 1 for a more detailed discussion of this point.

Moreover, the annular/circular OFTs are very suitable for the quality control of cylindrical polymer textile fibers and for detecting and rapid assessment of their optical inhomogeneities and/or local geometrical irregularities, which manifest themselves as deformations of the-anmular/circular interference fringes. An example is shown in Fig.13.

It has been stated that the OFTs of optical fibers ( see Figs.8 to 10) can be useful for qualification of these fibers and for a rapid assessment of their transverse birefringences. Some practical attempts to do this have been undertaken and some preliminary results will be published as a separate papers. At any rate, the interferometric system such as shown in Fig.11 appears to be more useful for this purpose than that shown in Fig. 1.

\section{1. coterusior}

The OFTs presented here appear to be interesting from both theoretical and practical points of view and show that a standard polarizing microscope only slightly modified and birefringent fibers constitute an attractive teaching tool for those who are interested in education in Fourier transform optics.

\section{REP EREACBS}

1. M.Pluta, "Optical Fourier transforms of birefringent fibers. I. Polymeric textile fibres." J.Modern Optics.34 (11), 1451-1470 ( 1987 ).

2. M.Pluta, "Appication of the aptical Fourier transform to metrolagy of polarizing lightguides." 2-nd Conference on Applications of lightguides, Bialowieza 1990, pp.106-117. (in Polish). 

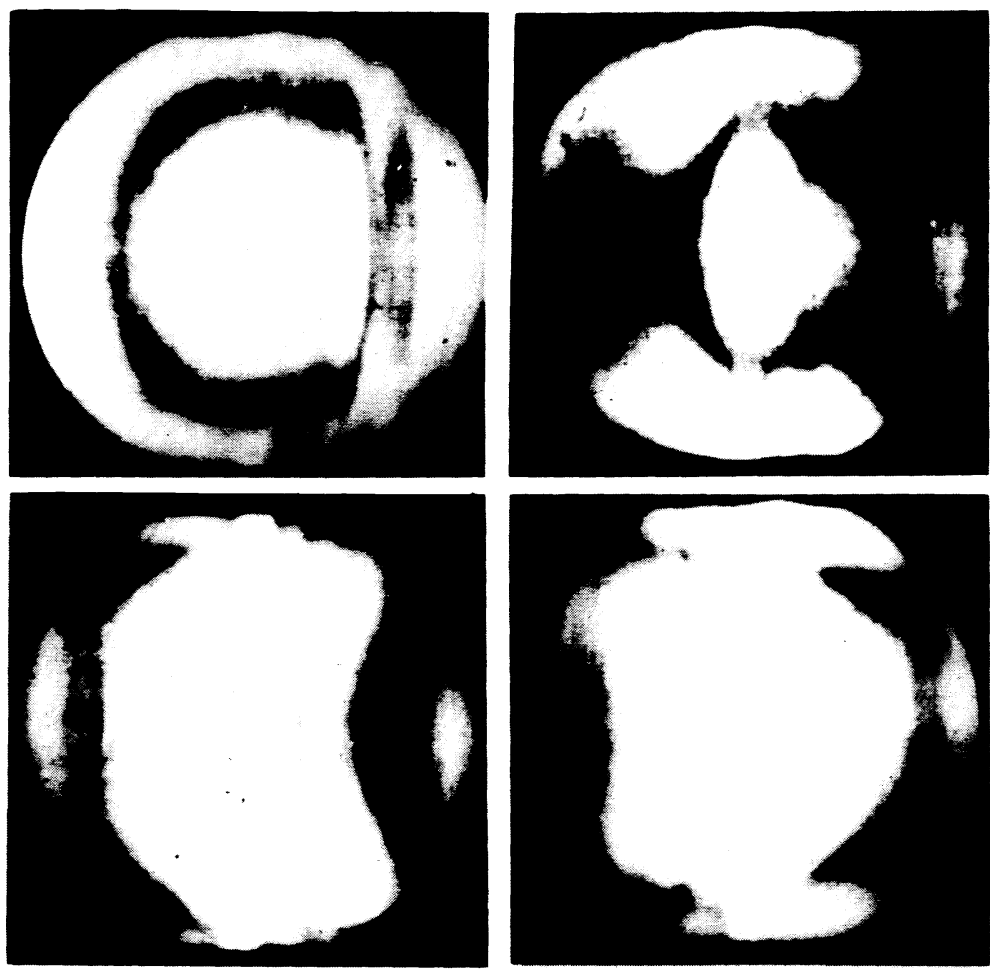

Fig.13. Irregular OFTs of a cyifindrical polyester textile fiber which suffers from local optical inhomogeneities and/or geometrical irregularities.

3. M.Plata, "Double refracting interferometer with variable direction of tilt of laterally sheared wavefronts." Optica Applicata 15 (1),77-89 (1985) .

4. M.Pluta, "Advanced Light Microscopy. Vol.T: Principles and Basic Properties, Amssterdam, and Polish Scientific Publishers ( PWN), Marsaw 1988, pp.379-394.

5. H.Bozyk, "Bxamination of physical parameters of polarizing lightguides by using polarized-light microinterferometry and optical Fourier transforms. Research Report DNS-T/05/102/90-2, Technical University of Blazystok, Institute of Mathematics and Physics, Białystok 1991 (in Polish). 